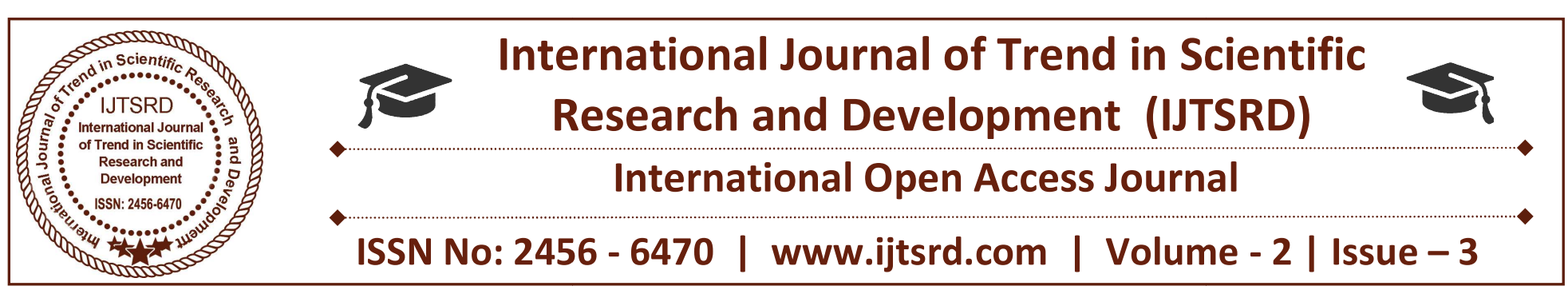

\title{
A Survey on Garbage Monitoring System using Internet of Things
}

\author{
V. Praveen, T. Arunprasad, R. Naveen, Dr. P. Gomathi \\ Department of CSE, N.S.N College of Engineering \& Technology, \\ Karur, Tamil Nadu, India
}

\begin{abstract}
The IoT Garbage Monitoring system is one of the innovative systems which will provides a healthy surroundings in the cities. The ultrasonic sensor is used for measuring the length of the bins, once the bins are being dumped with garbage the level of bins are shown graphically in the webpage. The data's are stored in the microcontroller and through the Wi-Fi module the data is being transferred to the webpage. The sensors are kept underneath the bins and once the garbage is being filled the data is transmitted to the microcontroller and from the microcontroller the data is regularly updated to the webpage through $\mathrm{Wi}-\mathrm{Fi}$ module, once the level of garbage in the bins reaches the fixed threshold value then there is a buzzer sound which indicates that the bin is full, the LCD display intimates about the bin which is filled and a LED light glows the data is regularly updated to the user monitoring it in the webpage. Thus this idea helps in maintaining a healthy environment.
\end{abstract}

Keywords: Ultrasonic Sensors; Wi-Fi; Microcontroller; LCD Display;

\section{Introduction}

A wide range of conventional family unit contraptions can be altered to work in an IoT framework. Wi-Fi network adapters, motion sensors, cameras, microphones and other instrumentation can be embedded in the devices to work in IoT. Home automation systems like light bulbs, other devices like wireless scales and wireless blood pressure monitors have been implemented already.

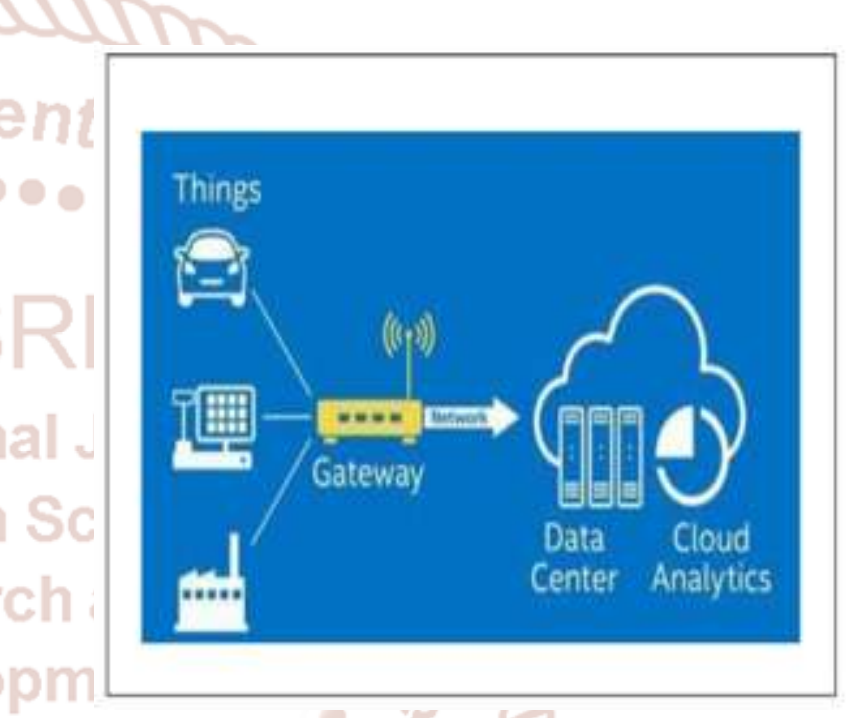

Fig.1 Working of IoT

IoT assumes that the underlying network equipment and the related technology can operate semiintelligently and automatically. Simply keeping mobile devices connected to the Internet will not make the devices to be smarter. Individuals have assorted necessities that require an IoT framework to adjust or be configurable for a wide range of circumstances and inclinations. Finally, even after overcoming all those challenges, if people become too reliant on this automation and the technology is not highly robust and any technical glitches in the system can cause serious physical and financial damage.

IoT assumes that the underlying network equipment and the related technology can operate semiintelligently and automatically. Simply keeping mobile devices connected to the Internet will not make the devices to be smarter. People have numerous needs that require an IoT gadget to adapt or 
be configurable for plenty special situations and preferences. Sooner or later, even after overcoming all the ones demanding situations, if people grow to be too reliant in this automation and the technology is not especially sturdy and any technical glitches inside the gadget can reason severe bodily and economic harm.

\section{Literature Survey}

1. Usually, in metropolis the rubbish bins or dustbins located at public locations are overloaded. It creates unhygienic conditions for humans as well as ugliness to that location leaving terrible odor. To keep away from such conditions there is an implementation of a proposed gadget known as IoT based totally smart garbage and Waste collection boxes.

2. Attributable to a paradigm shift toward IoT, researches into IoT offerings had been performed in a huge variety of fields. As a first-rate utility inside the subject of IoT, waste management has end up one such issue. The absence of efficient waste management precipitated extreme environmental troubles and price problems. the usage of IoT technology for waste control is one probably solution that waste discarded has been managed. it has been defined with the help of a simulation model. This version includes an Arduino controller, few garbage bins loaded with the sensors and monitored constantly thru an internet. The device additionally has a scope for citizen participation, in which any grievances from residents related to waste control is found.

3. Municipality takes many measures to preserve the cleanliness of the metropolis. One among which is organizing the dustbins in everyday distance for the convenience of public to discard the rubbish. Cleansing the garbage is a vital feature of municipality that is directly related to fitness issues. The version 'smart Dustbin' which indicates that the dustbin is filled to a certain level and cleaning or emptying them is a matter of instant difficulty. It prevents dumping of the garbage at the roadside dustbins which ends up giving the foul smell and the illness to people. The design of the clever dustbin includes a single directional cylinder and an Arduino Uno. The circuit to power up the mechanical gadgets is also embedded to acquire the preferred simulation.

4. With the growth in populace, the scenario of cleanliness with recognize to garbage control is degrading exceptionally. The overflow of rubbish in Public place creates the unhygienic situation within the surrounding. It may provoke numerous critical sicknesses amongst the close by humans. It also degrades the valuation of the region. To keep away from and to decorate the cleansing "clever garbage control system" is proposed. in the gadget, the extent of the rubbish within the dustbins is detected with the help of sensor systems, and communicated to the authorized manipulate room through GSM system. Microcontroller is used to interface the sensor gadget with GSM device. A Graphical User Interface (GUI) is also developed to display the favored information related to the rubbish for different decided on locations. This can help to control the rubbish series correctly.

5. The Smart Garbage Bins (SGB), which might be installed close to the condo homes and the man or woman homes, exchange facts with each different and send the statistics to the server through wireless verbal exchange. Structurally, the proposed machine is split into two domains: management domain and carrier area. Inside the management domain, information transferred from a SGB is analyzed and processed. In the service domain, resident throwaway their meals waste in a SGB, resident and SGB records is collected and transferred to the administration area.

6. The concept proposed saves the time for recycling and continues the earth inexperienced. The record consists of a way that how customers can effortlessly recycle the trash depending on steel or paper with the aid of the use of the trash basket. Clever computerized recycling trash basket will assist especially for folks who are workplace employee or college students because that is smaller than traditional one. The purpose of the proposed system is to basically create a recycling bin this is for college students and office workers who generally work or study sitting at the small size of the table, and they do now not want to spend a while to recycle metal or plastic cans every day. The maximum attractive point of the proposed device is that the existence of entire automatic motion. First, the principle lid of trash basket will act mechanically as some item procedures to the movement sensor. Second, the sorting plate will play a function to recycle whether the object is steel or paper. Third, once the trash basket is full, the capacity assessments 
Light Emitting Diode (LED) will warn to The webpage updation is done regularly for exchange the internal plastic bag. monitoring the level of garbage in bins and the intimation of the status of the bins is being regularly updated in the webpage once when the webpage is logged in by the user. The monitoring bins by this technique helps in keeping the surroundings clean. The use of microcontroller in this idea is ATmega32 as this microcontroller is being used for fast transmission of data and storage of data. There are many different microcontrollers in the family of AVR, but ATmega32 is used as the data is transferred fastly and much more efficiently than the other microcontrollers.

be done regularly in the smart cities by humans. To reduce the human intervention in monitoring the bins the use of IoT is introduced.

In the system, the level of the garbage in the dustbins is detected with the help of sensor systems, and communicated to the authorized control room through GSM system. Microcontroller is used to interface the sensor system with GSM system. A Graphical User Interface (GUI) is also developed to monitor the desired information related to the garbage for different selected locations. The use of GSM is not much efficiently used.

The garbage bins if not properly monitored then it may lead to various health issues, the problem is being solved by using the IR sensors which is being placed in the bins to detect whether the garbage is being filled or not, Once the garbage is full it will automatically send a message to the authorized person indicating that garbage is overloaded via GSM.' In particular, sending the level of garbage through GSM is more expensive, and the information sent through GSM may not receive to the authority persons at the right time to clean the garbage in the bins, so that the bins cannot be regularly monitored, by this the monitoring cannot be done much efficiently.

\section{Proposed System}

The proposed system monitors the rubbish bins and informs about the level of rubbish gathered inside the packing containers via a web page. Ultrasonic Sensors are used to feel the duration and level of the rubbish in the bins, and AVR own family of microcontroller is used to store the records of the ranges of the packing containers and the webpage is used for monitoring the bins from the place in which the consumer is being monitoring.

Ultrasonic sensors are used to detect the presence of targets and to measure the distance to targets in many automated factories and process plants. Sensors with an on or off virtual output are to be had for detecting the presence of objects, and sensors with an analog output which varies proportionally to the sensor to target separation distance are commercially available. The use of ultrasonic sensors in this idea is that it detects the object inside the bin and also measures the length of the bins and the data's are stored in the microcontroller, once any objects are thrown into the bins the sensors detects the object and intimates about the level of the bin in the webpage through the Wi-Fi module.

Wi-Fi is a technology for wireless local area networking.ESP8266 is a Wi-Fi module which is used for transmission of data using the wireless network it is more fast and efficient for transferring the data. The transfer rate of data depends upon the range and the distance. The area range of the network is also a reason for fast transmission of data. The Wi-Fi module is used for transmission of the data from the microcontroller to the webpage for the updation of webpage, the Wi-Fi module and the microcontroller is connected in the PCB board to exchange within themselves. ESP8266 is used for the fast transmission of data to the webpage. The Wi-Fi is used more efficiently and more accurately the data's are transferred to the webpage immediately when the net connection is established to it. 


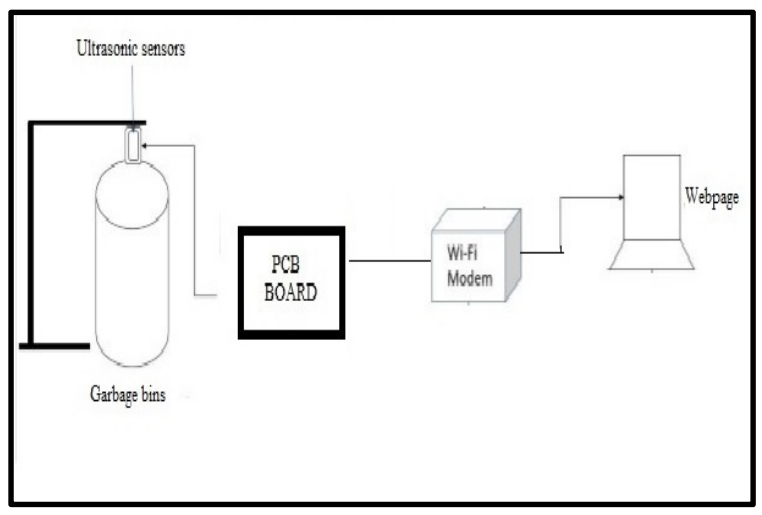

Fig.2 Garbage Monitoring System

LCDs are to be had to display arbitrary photos or constant photos with low records content, which can be displayed or hidden, such as pre-set phrases, digits, and seven-phase shows, as in a virtual clock. The use of LCD display is to display the details of bins that are connected by the ultrasonic sensors, the length of each bin is displayed in the display and the level of bins is also shown in number in the LCD display.

The data's that are collected are stored in the microcontroller (ATmega32) which is more efficient for the process of transmitting data to webpage through the Wi-Fi module all these hardware tools are connected in a PCB board.

It is an embedded circuit that interconnects all the hardware tools for maintaining the garbage level in the bins, and then the intimation of fully filled bins are indicated by a buzzer sound and a LED light glows by which the user maintaining webpage can immediately clean the garbage.

The intimation is done through the webpage, a buzzer sound and a LED light blinks in the kit by which this process will be useful for immediately cleaning the bins in the metropolitan cities.

This framework can be implemented in cities where there is lot of garbage which is not properly monitored and brings unhealthy issues, this technique can be used in various places where the garbage is to be monitored, it can be used by any common man because the intimation to the user is shown graphically which can be understood by the illiterates also, so this technique is beneficial to all common man.

\section{RESULTS AND DISCUSSION}

The proposed experimental results uses the ultrasonic sensors which senses the depth of the bins and updates the level of dump in the bins to the user monitoring it, the values are updated in the webpage, the levels of bins are also shown in the LCD display, the webpage is designed in a diagrammatic so that it will be more convenient for the user monitoring it.

Therefore the intimation will be shown in the webpage and a LED light blinks in the PCB board and a buzzer sound occurs so that the user will be intimated immediately and the garbage in the bins will be cleaned by the people working in government sectors, by this the cities can be kept clean.

This proposed system can be implemented Hospitals, Government sectors, municipality, etc. This proposed system helps in monitoring the dumped garbage bins regularly.

\section{CONCLUSION}

The survey done on this Garbage Monitoring System is that the bins are being monitored regularly by the user through the webpage in which the level of the bins are indicated and once the bin is being filled up by the dump and through the sensor kept underneath the bin, the intimation of the filled up bins are given by a buzzer sound and a LED light blinks which gives a immediate intimation to the user monitoring it, in this survey the additional features that can be added is that during the measurement of bin the use of IR sensor can be used.

\section{REFERENCES}

1. Abhishek Dev, Maneesh Jasrotia, Muzammil Nadaf, Rushabh Shah. "IoT Based Smart Garbage Detection System", International Research Journal of Engineering and Technology (IRJET) Volume: 03, Issue: 12, December 2016.

2. Meghana K C, Dr. K R Nataraj, "IoT Based Intelligent Bin for Smart Cities", International Journal on Recent and Innovation Trends in Computing and Communication, IJRITCC, May 2016.

3. Twinkle Sinha, K.Mugesh Kumar, P.Saisharan, "Smart Dustbin" International Journal of Industrial Electronics and Electrical Engineering, ISSN: 2347-6982 Volume-3, Issue-5, May 2015. 
International Journal of Trend in Scientific Research and Development (IJTSRD) ISSN: 2456-6470

4. Vikrant Bhor, Pankaj Morajkar, Maheshwar Gurav, Dishant Pandya,"Smart Garbage Management System", International Journal of Engineering Research \& Technology (IJERT)ISSN:2278-0181, Vol. 4 Issue 03, March 2015.

5. Insung Hong, Sunghoi Park, Beomseok Lee, Jaekeun Lee, Daebeom Jeong, and SehyunPark,"IoT-Based Smart Garbage System for Efficient Food Waste Management”, School of
Electrical and Electronics Engineering, ChungAng University, Seoul 151-756, Republic of Korea, August 2014.

6. Suwon Shin, Kaiyuan Fan, "Smart Automatic Recycling Trash Basket", Final Report for ECE 445, Senior Design, fall 2012 TA: Lydia Lee Majure, 12, December 2012.

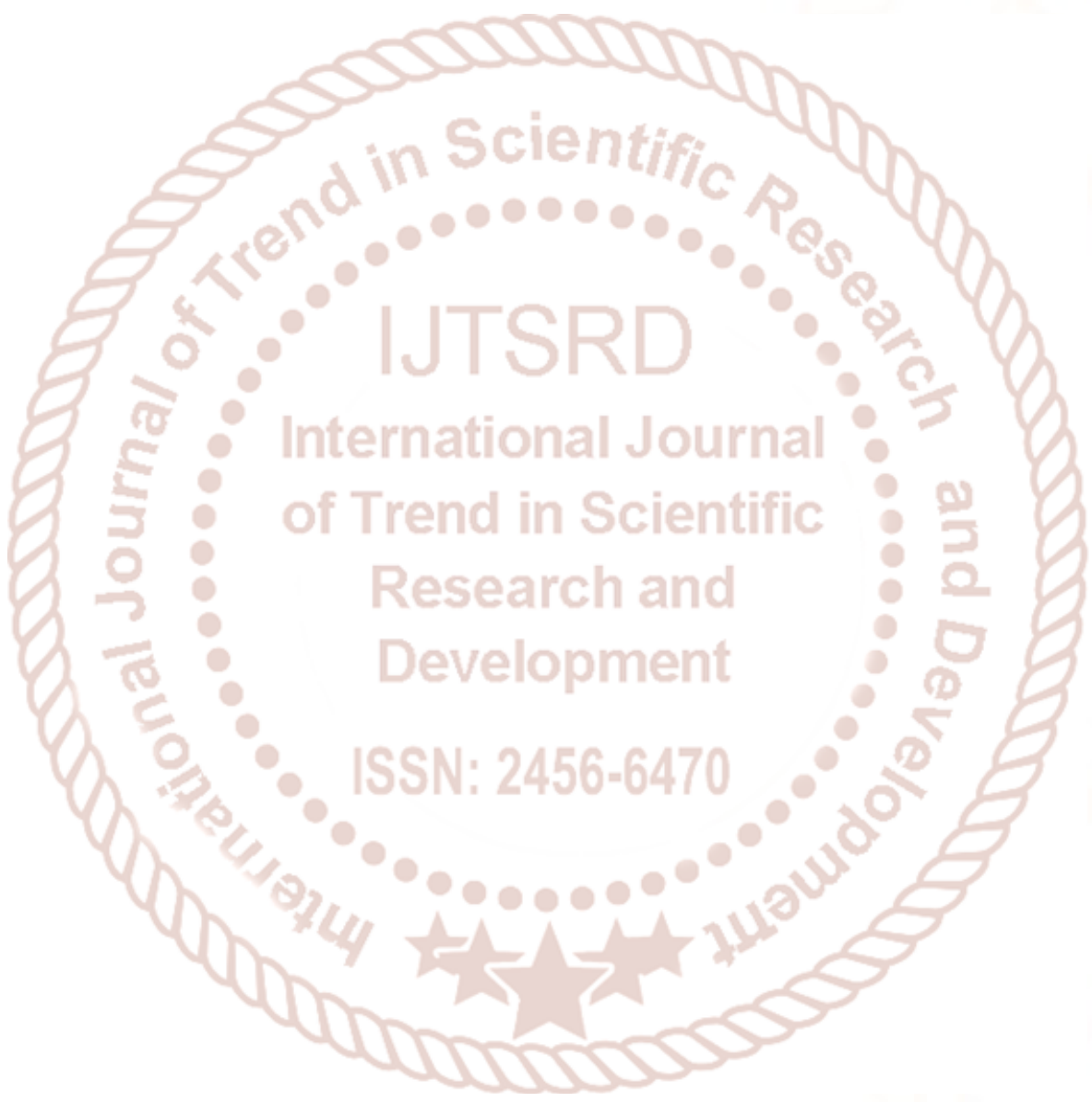

